\title{
Design and research of home security patrol robots
}

\author{
Gai Kerong ${ }^{1, a^{*} \text {, Zhou Fengying }}{ }^{2, b}$ and Zhao Linshan ${ }^{3, c}$ \\ Beijing Polytechnic College, No.368 Shimen Road, Shijingshan District Beijing 100042,china \\ Beijing Polytechnic College, No.368 Shimen Road, Shijingshan District Beijing 100042,china \\ School of Mechanical Engineering\&Autumation of BUAA,No.37 Xueyuan Road Beijing \\ 100191,china \\ gkr0706@126.com,niuxt@163.com, zls1007@163.com
}

Keywords: security inspection, robot, omnidirectional movement, remote monitoring

Abstract. With a growing trend of social aging, the issue of "empty-nest" elderly is increasingly serious and children are more eager to keep an eye on the living security of the elderly in real time. In light of the increasing demand on home security monitoring, the real-time performance of existing security monitoring products, privacy and the users "sense of being watched" and other issues, this paper introduces the design of a home security patrol robot prototype which realizes automatic obstacle avoidance, security inspection, automatic charging and remote control and other key technical indicators. In the on-site inspection, the prototype system has shown excellent robustness and flexibility while meeting the designated indicators

\section{Introduction}

With a growing trend of social aging and accelerated pace of life, it becomes increasingly common for children and the elderly to live in different cities and the issue of "empty-nest" elderly becomes increasingly serious. In order to ensure security of the elderly, it needs to implement real-time monitor to the home security. With rapid development of the computer technology, image processing technology, mobile Internet technology and robot technology, it is of great significance to develop a home security patrol robot ${ }^{[1]}$ integrating monitoring, alarming, education, entertainment, and even assistance and home care of the elderly to improve the living quality and materialize the concept of the smart city.

\section{General design}

The general design plan of the security patrol robot is shown in Fig. 1. It consists of the chassis motion unit, multi-sensor obstacle avoidance unit, smart terminal control unit, visual positioning and navigation unit and automatic charging unit. The robot can move flexibly in the home environment, automatically avoid obstacles, and make real-time monitoring to the smoke and temperature and other security indicators of the house and display such data at the smart terminal and control the indicators through the terminal.

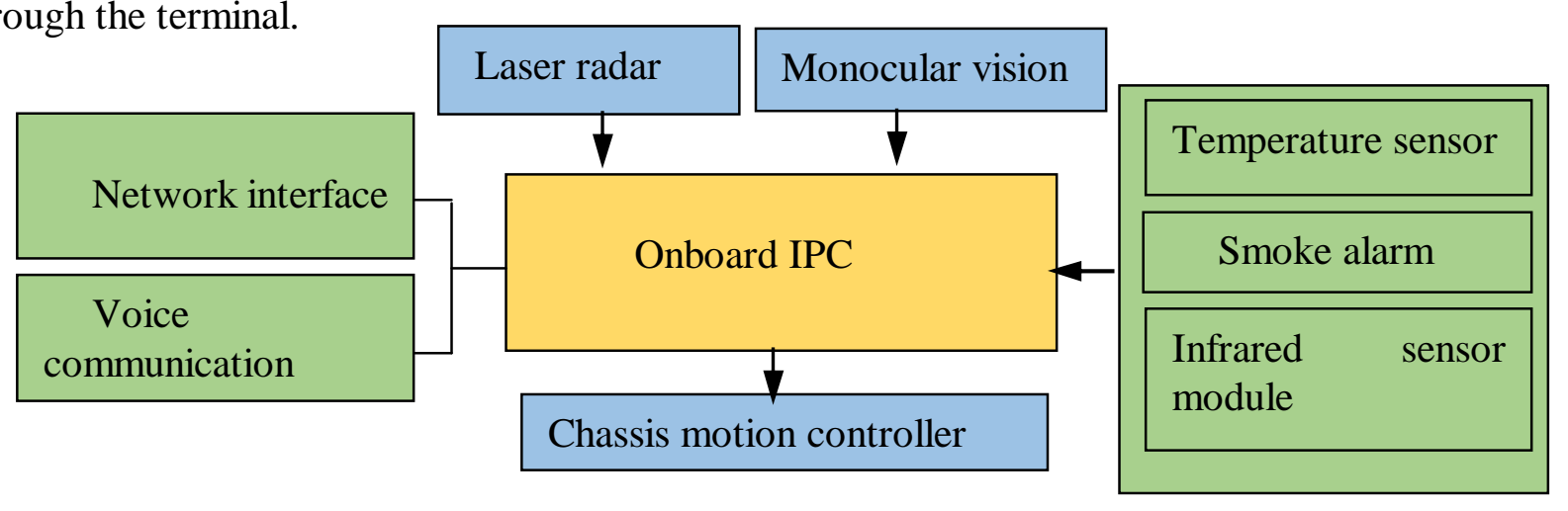

Fig. 1 General design of the system 


\section{Chassis motion unit}

The aisle in the home environment is normally as wide as allowing a person to pass through and some furniture are relocated from time to time. Therefore the environment is normally complicated. In light of such characteristics, the design of the robot's mechanical structure should observe the principle of realizing movement flexibility and good trafficability, and adopt the omnidirectional wheels that can run freely in narrow space to realize omnidirectional movement ${ }^{[2,3]}$. The robot chassis adopts the structure consisting of three omnidirectional wheels, a servo motor and a photoelectric encoder. The three Swedish wheels are installed radially symmetrically and form a $120^{\circ}$ angle with each other. The crossing point of axes coincide with the robot's center of gravity and the driven wheel is perpendicular to the capstans ${ }^{[4,5]}$. When the chassis rotates in situ, and the direction of all omnidirectional capstans is perpendicular to the ligature between the chassis rotating center and the wheel center, the internal resultant force of the system is zero and the driving force of system reaches the optimal effect.

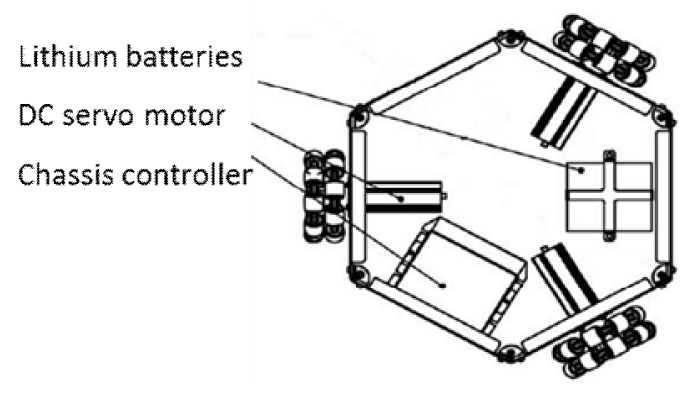

Fig. 2 Three-wheel omnidirectional chassis

The robot chassis adopts the DC servo motor driving system and a retarder with transmission ratio at 64 is installed between the servo motor and the omnidirectional wheels. The chassis uses the external bearing block deign and the axial and radial load delivered by wheels is borne by bearing in the bearing block. The motor and retarder are used to transmit torque.

The hardware of the chassis motion control system consists of the motion control unit, upper motion planning unit, bottom obstacle avoidance unit, power and level switch unit. The motion control unit ${ }^{[6 \text {, }}$ ${ }^{7]}$ is the key to realize controllable motion of the robot. It consists of the control part and driving part. The control part ${ }^{[8]}$ is mainly used to receive the control signals from the host computer and feedback signals from the chassis by performing corresponding kinetics algorithm and logical judgment. The control part uses STM32 processor and ARM system of Harvard architecture, with rich external interface circuit. The driving part is mainly used to realize smooth movement of the servo motor. It consists of three sets of DC servo motors and corresponding drivers and can realize precise and sensitive movement of the motor.

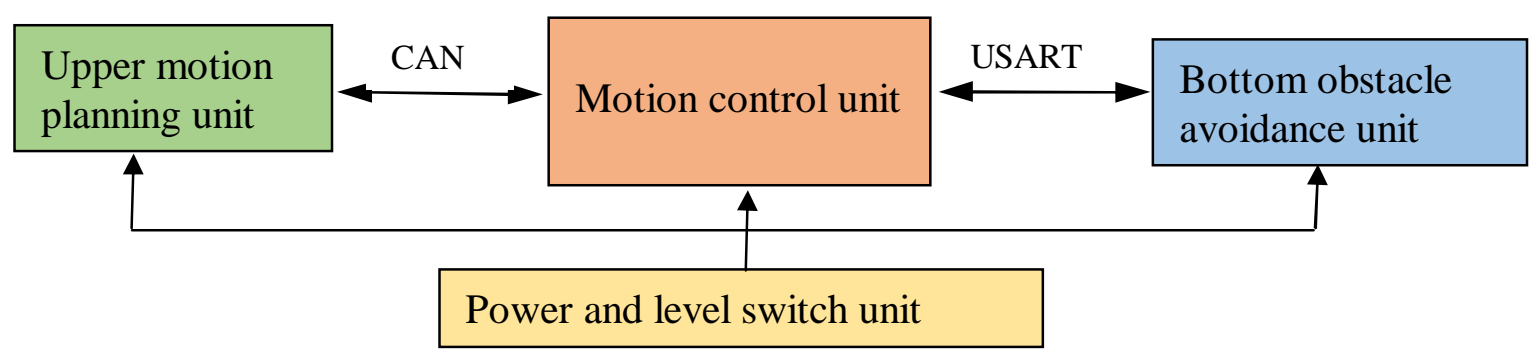

Fig. 3 Frame diagram of the chassis motion unit

The software for the bottom control system consists of 1) motion command analytic program, 2) odometer attitude feedback program, and 3) collision detection and error handling program. The relations among them are shown in Fig. 4. 


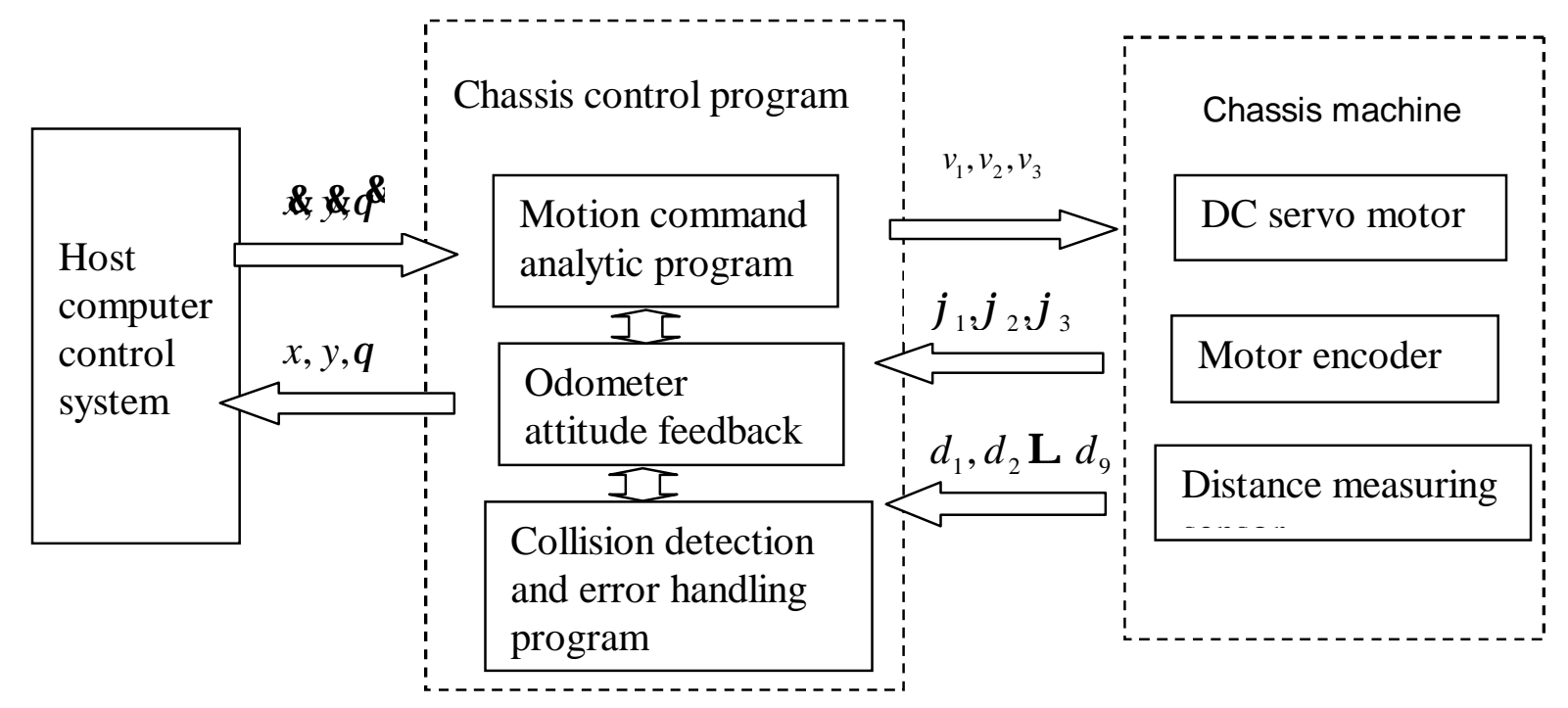

Fig. 4 Relations among various parts of the chassis control software system Of that, $\mathrm{x}, \mathrm{y}$ and $\theta^{\mathcal{E}}$ are the translational velocity and the rotation angular velocity; $v_{1}, v_{2}, v_{3}$ are the angular velocity of each of the omnidirectional wheel; $x, y, \theta$ are the changed value of displacement and attitude of the chassis motion; $\varphi_{1}, \varphi_{2}, \varphi_{3}$ are the angle that the omnidirectional wheels have rotated; $d_{1}, d_{2} \ldots . d_{9}$ are the distance to the obstacles detected by the distance measuring sensor.

Fig. 5 offers the definition of the chassis motion coordinate system: of that, $X_{1} O_{1} Y_{1}$ is the overall coordinate system of the robot, which directly decides the location and attitude of the robot in the room; $\mathrm{X}_{2} \mathrm{O}_{2} \mathrm{Y}_{2}$ is the body-fixed coordinate system of the robot, and the forward direction of the x-axis is the front of the robot and keeps relatively static when the robot is in motion; $\theta$ is the included angle between the overall coordinate system and the body-fixed coordinate system, deciding the attitude of the robot; $v_{1}, v_{2}, \quad v_{3}$ are the angular velocity of rotation of each omnidirectional wheel; $L$ is the distance between the center of the omnidirectional wheels and the chassis center, subject to the mechanical structure of the robot; the kinetics equation of the three-wheel omnidirectional chassis is as follows:

$$
\left[\begin{array}{l}
v_{1} \\
v_{2} \\
v_{3}
\end{array}\right]=\left[\begin{array}{ccc}
-\sin \left(\frac{\pi}{3}+\theta\right) & \cos \left(\frac{\pi}{3}+\theta\right) & L \\
\sin \theta & -\cos \theta & L \\
\sin \left(\frac{\pi}{3}-\theta\right) & \cos \left(\frac{\pi}{3}-\theta\right) & L
\end{array}\right]\left[\begin{array}{l}
x \\
\alpha \\
\theta
\end{array}\right]
$$

The odometer attitude feedback program accumulates the motion of the omnidirectional wheels to get the data of displacement and attitude of the robot in actual motion. The kinetic equation is as follows:

$$
\left[\begin{array}{l}
\alpha \\
\Leftrightarrow \\
\theta
\end{array}\right]=\left[\begin{array}{ccc}
-\sin \left(\frac{\pi}{3}+\theta\right) & \cos \left(\frac{\pi}{3}+\theta\right) & L \\
\sin \theta & -\cos \theta & L \\
\sin \left(\frac{\pi}{3}-\theta\right) & \cos \left(\frac{\pi}{3}-\theta\right) & L
\end{array}\right]^{-1}\left[\begin{array}{l}
\phi \alpha \\
\alpha \\
\alpha
\end{array}\right]
$$

With the kinetic equations mentioned above, we can get the attitude increment of the robot chassis and accumulate the increment to finally get the real attitude of the robot. When the calculation interval is 
small enough, the error can be controlled to the engineering permissible range. In this system the calculation frequency is $1000 \mathrm{~Hz}$.

\section{Multi-sensor integrated obstacle avoidance unit}

In order to avoid various obstacles in the ambient environment, the robot chassis should automatically avoid various obstacles in motion. The obstacle avoidance part consists of the bottom and the upper obstacle avoidance modules. The upper obstacle avoidance module is to locate the obstacle by using the laser radar and monocular vision to get the information of the ambient environment and automatically avoid the obstacles when planning the path. The bottom obstacle avoidance module mainly completes this process with the ultrasonic distance sensor group installed on the omnidirectional chassis. Nine ultrasonic distance sensors are used according to the shape of the chassis. The sensors and the bottom processor communicate with each other through the serial port. See Fig. 6 for the specific installation position of the sensors. The ultrasonic sensors are mainly used for the long-distance obstacle avoidance, with the laser radar used in coordination. The axis sensing model and the filtering method of tolerance function are used to process this task. The laser radar obstacle avoidance strategy uses $\mathrm{VHF}^{[9]}$ method to avoid obstacles by using grids to represent the environment. The data obtained with these two kinds of sensors will be integrated with the adaptive method. When the distance is far, the ultrasonic data will be given higher weight while when the distance is near and the laser radar data will be given higher weight.

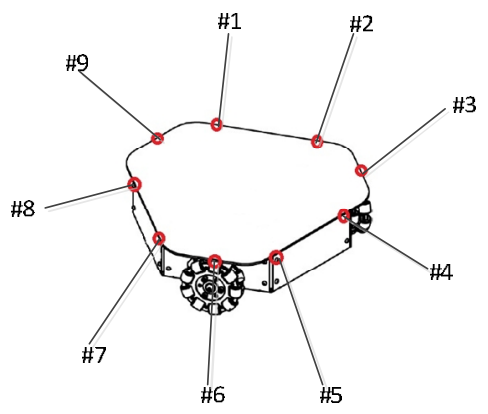

Fig. 5. Map of installation position of ultrasonic measuring sensors

The hardware structure of the upper motion planning unit is mainly a high-performance industrial computer. It receives laser radar signals to structure the environmental map for the robot path planning and obstacle avoidance. It receives data from the monocular panoramic camera and displays the image on the visual interface to allow the user to observe and inspect the ambient environment of the robot and "tell" the system to move to the designated position as a part of the interactive entry.

The robot can realize excellent self-location through visual sense ${ }^{[10]}$. Fig. 7 shows an imitated ordinary family environment layout. On the right diagram, the visual tags are put at the places with star on the ceiling on the left diagram. See Fig. 8 for the visual tag navigation flow. The robot captures the pictures on ceiling with monocular vision, and extracts the tag characteristics from Opencv library so as to realize the self-location. When the robot enters the roaming status, it will "get lost". At this moment, the identification of the visual tags can allow the robot to rapidly reach the designated position.

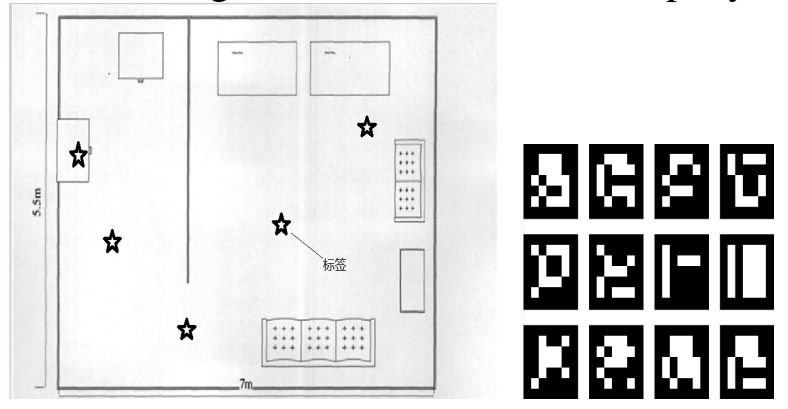

Fig. 6. Modelling of ordinary family environment layout and visual tags 


\begin{tabular}{|c|c|c|}
\hline $\begin{array}{l}\text { Monocular vision captures } \\
\text { the information of images } \\
\text { on the ceiling }\end{array}$ & $\begin{array}{l}\text { Identifying the tags } \\
\text { from the Opencv } \\
\text { library }\end{array}$ & $\begin{array}{l}\text { Analyzing the tags, and } \\
\text { obtains the location of } \\
\text { the robot }\end{array}$ \\
\hline
\end{tabular}

Fig. 7. Visual tag identification and navigation flow

\section{Automatic charging system}

The robot adopts the underneath charging mode with the charge stand. This system drives the robot with eight AA Nickel-cadmium batteries and the machine can reach the charge stand from any direction within the range of $180^{\circ}$. After checking location, the robot starts battery charge and automatically step down the charge stand after completing recharging. As the laser radar has a short period of distance measuring and good real-time performance, the robot uses the time varying potential field of multiresolution to set up the environment model and describe the dynamic environment where the robot is in and plans the movement path of the robot with the fuzzy control to finally realize precise automatic charge.

\section{Smart terminal control unit}

As shown in Fig. 9, the smart terminal control unit consists of the robot remote control module, monitoring video real-time display module, security indicator display module and security alarm module. The main functions include

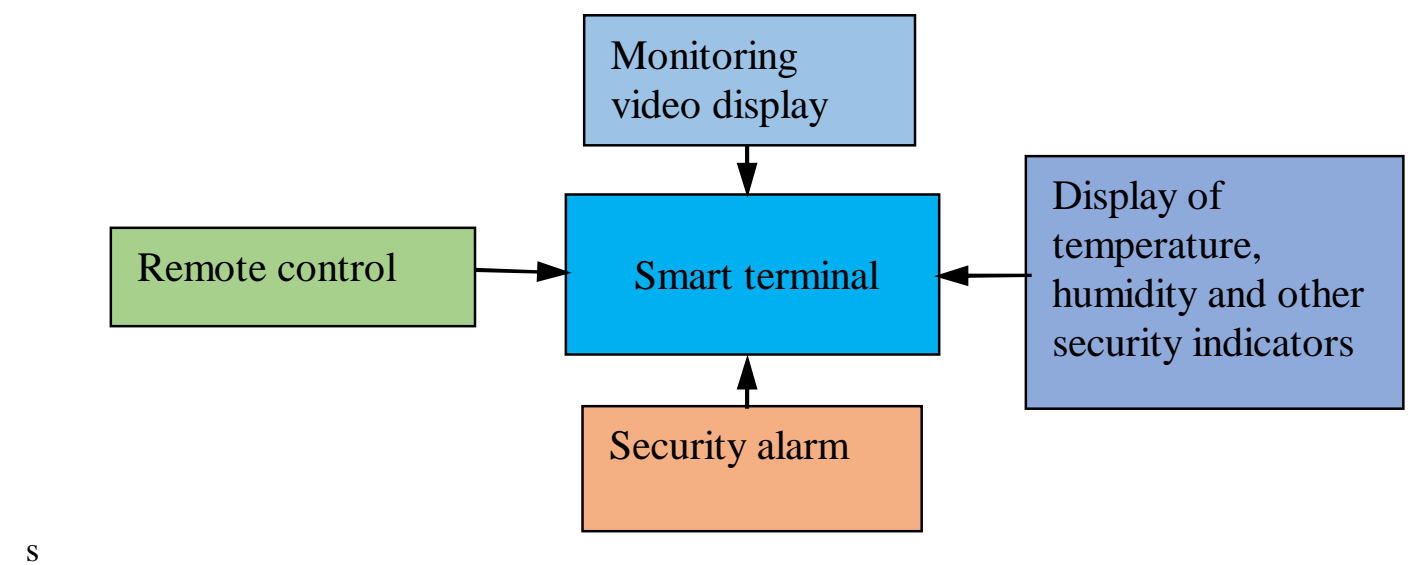

Fig. 8. Schematic diagram of smart terminal

1. Real-time display of the monitoring video collected by the robot with the monocular vision at the remote smart terminal;

2. Real-time display of the security indicators such as temperature, humidity and gas concentration collected by the robot with sensors at the remote smart terminal;

3. Remote control of the robot with the remote smart terminal;

4. Signal alarms at the remote smart terminal when the gas concentration and other security indicator exceeds the designated threshold value.

The robot is installed with a WIFI module. The system is connected with Internet with the smart terminal through the data service provided by the mobile communication network or WIFI module to realize the data communication of the two parts. The user can connect with the robot through a mobile phone. See Fig. 10 for the remote control with mobile phone. The user can watch the video collected by the robot camera to have an intuitive understanding to the environment and control the robot with the buttons on interface of the mobile phone to move forwards, backwards or turn left or right or control the switch of the LED light on eyes of the robot to clearly observe the situation of the house. The server and smartphone client end use the UDP protocol to ensure the continuity of video transmission and solve the problem of time delay in the remote control. 

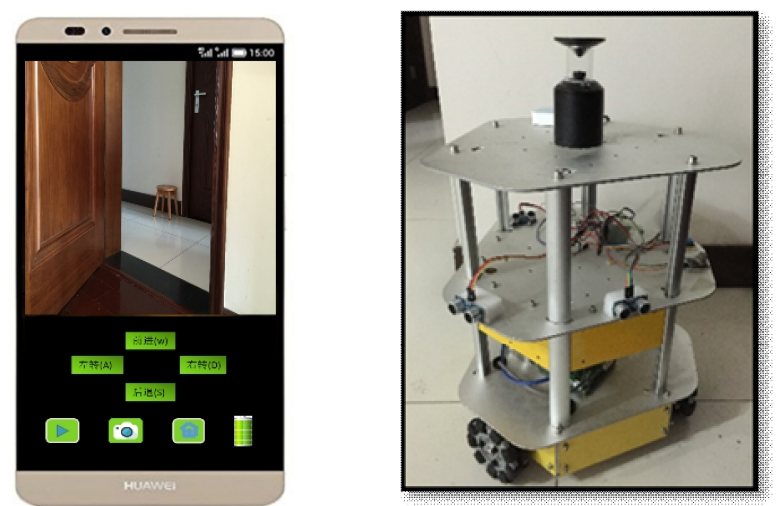

Fig. 9 Mobile phone remote control and prototype

\section{Conclusion}

This paper introduces the design of multi-sensor information system based home security patrol omnidirectional mobile robot. With the establishment of the three-wheel omnidirectional model, the robot can realize control of movement in any direction, and high-efficient obstacle avoidance with integrated processing of the ultrasonic and laser radar sensors, and real-time remote object image display and mobile control with the Internet-based control and machine vision, and monitoring and alarm of daily security of the house with the sensors of gas, smoke and temperatures.

\section{References}

[1] Marini C P, Ritter G, Sharma C, et al. The effect of robotic telerounding in the surgical intensive care units impact on medical education [J]. Journal of Robotic Surgery, 2015, 9(1): 51-56.

[2] Ma S, Ren C, Ye C. An omnidirectional mobile robot: Concept and analysis[C]//Robotics and Biomimetics (ROBIO), 2012 IEEE International Conference on. IEEE, 2012: 920-925.

[3] Savkin A V, Hoy M. Reactive and the shortest path navigation of a wheeled mobile robot in cluttered environments [J]. Robotica, 2013, 31(02): 323-330.

[4] Ren C, Ma S. Dynamic modeling and analysis of an omnidirectional mobile robot[C]//Intelligent Robots and Systems (IROS), 2013 IEEE/RSJ International Conference on. IEEE, 2013: 4860-4865.

[5] Indiveri G. Swedish wheeled omnidirectional mobile robots: kinematics analysis and control [J]. IEEE transactions on robotics, 2009, 25(1): 164-171.

[6] Li S A, Lo C H, Weng C W, et al. Design of the wheeled robot motor controller[C]//Intelligent Signal Processing and Communications Systems (ISPACS), 2012 International Symposium on. IEEE, 2012: 250-253.

[7] Viet T D, Doan P T, Hung N, et al. Tracking control of a three-wheeled omnidirectional mobile manipulator system with disturbance and friction[J]. Journal of mechanical science and technology, 2012, 26(7): 2197-2211.

[8] Fontanelli D, Greco L, Palopoli L. Soft real-time scheduling for embedded control systems [J]. Automatica, 2013, 49(8): 2330-2338.

[9] Cai zi xing, Zhengmingjie, Zouxiaobing. Real-time obstacle avoidance for mobile robots strategy basedon laser radar [J]. Journal of Central South University, 2006,37（2） : 324-329.

[10] Yu Jinxia, Wang Lu, Cai Zixing, Self-location of Mobile Robot in Unknown Environment [M] . Beijing, Publishing House of the Electronics Industry, 2011. 